\title{
MAGNETOOPTICS OF III-V COMPOUNDS IN MANY BAND MODEL APPROACH *
}

\author{
A. OBuchowicz and J. Wlasak \\ Institute of Physics, Technical University of Wrocław, Wyb. Wyspiańskiego 27, \\ 50-370 Wrocław, Poland
}

(Received August 8, 1990)

\begin{abstract}
III-V semiconducting compounds in the presence of a magnetic field are considered. The $14 \times 14$ effective hamiltonian in the frame of 5-level model is obtained. Directional dependence of selection rules are obtained. Shallow donor levels in the frame of spherical 3-level model are also considered. New trial functions suitable for a whole range of magnetic field are proposed. It is found that the energy as well as the variational function of each magnetodonor level is a continuous function of the magnetic field.
\end{abstract}

PACS numbers: 78.20.Ls, 61.50.Em, 71.55.-i

The majority of $\mathrm{kp}$ calculations of a semiconductor band structure follow the procedure of perturbation theory for nearly degenerate levels (Löwdin [1]): one includes a small number of bands in matrix equation and treats the other bands in perturbation theory. Such matrix for $\Gamma_{6}, \Gamma_{7}$ and $\Gamma_{8}$ bands in III-V semiconductors in the presence of magnetic field in [001] direction was introduced by Pidgeon and Brown [2] and consequently extended to other magnetic field directions by Weiler et al. [3], Brown and Rössler [4], Wlasak [5] and Grisar et al. [6].

The present paper extends construction of this matrix. We have additionally taken into account $\Gamma_{7}$ and $\Gamma_{8}$ conduction bands, thus we obtain $14 \times 14$ matrix of effective hamiltonian for arbitrary direction of magnetic field. The $Q$-depended part of our hamiltonian (where $Q=-(i \hbar / m)<X^{v}\left|p_{y}\right| Z^{c}>$ ) reduces to the forms presented by Pfeffer and Zawadzki [7] for the three directions they have considered (i.e. [001], [110] and [111]). Matrix elements of magnetooptical transitions are calculated in the standard way [6]. Selection rules for inversion asymmetry induced transitions are the same as those in the paper of Wlasak [8]. The angle dependence of selection rules for 'warping' induced transitions is summarized in Table I. The symbol $\left(k_{H}\right)$ in this Table denotes both warping and $k_{H} \neq 0$ induced transitions.

*This work has been partially supported by CPBP 01.06./9.02. 
We denote by $\Delta s=0$ the transitions within the $a$ set or the $b$ set of solutions, by $\Delta s=-1$ the transitions from the $a$ set to the $b$ set and by $\Delta s=+1$ the transitions from the $b$ set to the $a$ set in the Pidgeon and Brown [2] notation. $\sigma_{\mathrm{L}}$ and $\sigma_{\mathrm{R}}$ describe left and right circular polarizations in the Faraday geometry, respectively and $\pi$ describes parallel Voigt geometry.

TABLE I

The angle dependence of selection rules for warping induced transitions

$$
\begin{gathered}
\beta_{0}=-s^{2}\left(1+3 c^{2}\right)+s^{4} \cos ^{2} 2 \phi ; \\
\beta_{1}=s \exp (\mathrm{i} \psi)\left[c\left(1-3 c^{2}\right)+s^{2} c \cos ^{2} 2 \phi+0.5 \mathrm{i} s^{2} \sin 4 \phi\right] ; \\
\beta_{2}=s^{2} \exp (2 \mathrm{i} \psi)\left[\left(1-3 c^{2}\right)-\left(1+c^{2}\right) \cos ^{2} 2 \phi-\mathrm{i} c \sin 4 \phi\right] ; \\
\beta_{3}=s \exp (3 \mathrm{i} \psi)\left[c\left(5-3 c^{2}\right)-c\left(c^{2}+3\right) \cos ^{2} 2 \phi-0.5 \mathrm{i}\left(1+3 c^{2}\right) \sin 4 \phi\right] ; \\
\beta_{4}=\exp (4 \mathrm{i} \psi)\left\{\left[4 s^{4}-\left(1+c^{2}\right)^{2}\right]+\left[4 s^{4}+\left(1+c^{2}\right)^{2}\right] \cos ^{2} 2 \phi+\right. \\
\left.+\mathrm{i} c\left(1+c^{2}\right) \sin 4 \phi\right\}
\end{gathered}
$$

$s$ and $c$ denote $\sin \theta$ and $\cos \theta$, respectively,

$\psi, \phi, \theta$ are Euler's angles measured from the [001] direction.

\begin{tabular}{r|r|ll|llll}
\hline \hline \multirow{2}{*}{ funct. } & \multirow{2}{*}{$\Delta s$} & \multicolumn{3}{|c|}{$\sigma_{\mathrm{R}}$} & \multicolumn{2}{|c}{$\sigma_{\mathrm{L}}$} & \multicolumn{2}{c}{$\pi$} \\
\cline { 3 - 7 }$\left|\beta_{0}\right|^{2}$ & -1 & $0\left(k_{H}\right)$ & & $+2\left(k_{H}\right)$ & +1 & \\
& 0 & -1 & & +1 & & $0\left(k_{H}\right)$ \\
& +1 & $-2\left(k_{H}\right)$ & & $0\left(k_{H}\right)$ & -1 & \\
\hline \multirow{3}{*}{$\left|\beta_{1}\right|^{2}$} & -1 & -1 & $;+1$ & +1 & $;+3$ & $0\left(k_{H}\right)$ & $;+2\left(k_{H}\right)$ \\
& 0 & $-2\left(k_{H}\right)$ & $; 0\left(k_{H}\right)$ & $0\left(k_{H}\right)$ & $;+2\left(k_{H}\right)$ & -1 & $;+1$ \\
& +1 & -3 & $;-1$ & -1 & $;+1$ & $-2\left(k_{H}\right)$ & $; 0\left(k_{H}\right)$ \\
\hline \multirow{3}{*}{$\left|\beta_{2}\right|^{2}$} & -1 & $-2\left(k_{H}\right)$ & $;+2\left(k_{H}\right)$ & $0\left(k_{H}\right)$ & $;+4\left(k_{H}\right)$ & -1 & $;+3$ \\
& 0 & -3 & $;+1$ & -1 & $;+3$ & $-2\left(k_{H}\right)$ & $;+2\left(k_{H}\right)$ \\
& +1 & $-4\left(k_{H}\right)$ & $; 0\left(k_{H}\right)$ & $-2\left(k_{H}\right)$ & $;+2\left(k_{H}\right)$ & -3 & $;+1$ \\
\hline \multirow{2}{*}{$\left|\beta_{3}\right|^{2}$} & -1 & -3 & $;+3$ & -1 & $;+5$ & $-2\left(k_{H}\right)$ & $;+4\left(k_{H}\right)$ \\
& 0 & $-4\left(k_{H}\right)$ & $;+2\left(k_{H}\right)$ & $-2\left(k_{H}\right)$ & $;+4\left(k_{H}\right)$ & -3 & $;+3$ \\
& +1 & -5 & $;+1$ & -3 & $;+3$ & $-4\left(k_{H}\right)$ & $;+2\left(k_{H}\right)$ \\
\hline \multirow{2}{*}{$\left|\beta_{4}\right|^{2}$} & -1 & $-4\left(k_{H}\right)$ & $;+4\left(k_{H}\right)$ & $-2\left(k_{H}\right)$ & $;+6\left(k_{H}\right)$ & -3 & $;+5$ \\
& 0 & -5 & $;+3$ & -3 & $;+5$ & $-4\left(k_{H}\right)$ & $;+4\left(k_{H}\right)$ \\
& +1 & $-6\left(k_{H}\right)$ & $;+2\left(k_{H}\right)$ & $-4\left(k_{H}\right)$ & $;+4\left(k_{H}\right)$ & -5 & $;+3$
\end{tabular}

Let us now consider shallow magnetodonors in III-V compounds. The use of 3-level model to solve this problem began from the work of Larsen [9] and was developed by Zawadzki and Wlasak [10] and Trzeciakowski et al. [11]. The present calculations are performed in the frame of this model.

We have taken trial functions for the ground and excited states in the following form

$$
\begin{gathered}
f_{1 s}=C_{1 s} \exp \left(-\beta r^{2 \delta}\right), \\
f_{p m}=C_{p m} r^{2 \delta} \exp \left(-0.5 \beta r^{2 \delta}\right) \sin \theta \exp (\mathrm{i} m \phi), m= \pm 1, \pm 2 \ldots \\
f_{d m}=C_{d m} r^{4 \delta} \exp \left(-0.5 \beta r^{2 \delta}\right) \sin \theta \cos \theta \exp (\mathrm{i} m \phi), m= \pm 1, \pm 2 \ldots \ldots
\end{gathered}
$$

where: $r=\left(\rho^{2}+\lambda z^{2}\right)^{0.5} ; \beta=0.5(\gamma \varepsilon / 2 \lambda)^{\delta} ; \lambda, \varepsilon$ and $\delta$ are variational parameters. One can see that for $\delta=0.5$ and $\lambda=1$ Eq.(1) approaches $1 s$ hydrogenic function 
and for $\delta=1$ it is the high field function of Yafet et al. [12]. For $m= \pm 1, \delta=0.5$ and $\lambda=1$ Eq.(2) gives simply $2 p_{x}$ and $2 p_{y}$ functions. The series in Eq.(3) originates from $3 d_{x+z}$ and $3 d_{y+z}$ functions. The introduction of the parameter $\delta$ gives the possibility of using our functions over the whole range of magnetic field.

Energy difference between excited and ground states in $\mathrm{cm}^{-1}$.

TABLE II

Excited states are described in terms of functions (2) and (3).

\begin{tabular}{c|r|r|r|r|r|r|r|r|r}
\hline \hline Com- & Mag. & \multicolumn{6}{|c}{$p m$} & \multicolumn{4}{|c}{ final states } \\
\cline { 3 - 9 } pound & Field & \multicolumn{4}{|c}{$p m$} & \multicolumn{4}{|c}{$p d$} \\
\cline { 3 - 9 } & {$[\mathrm{kGs}]$} & $m=-1$ & $m=1$ & $m=2$ & $m=3$ & $m=-1$ & $m=1$ & $m=2$ & $m=3$ \\
\hline InAs & 1 & 7.3 & 11.3 & 17.0 & 21.7 & 9.9 & 13.9 & 18.4 & 22.8 \\
& 10 & 9.1 & 48.1 & 88.8 & 128.1 & 18.9 & 57.8 & 95.1 & 133.1 \\
& 30 & 12.9 & 125.8 & 235.0 & 339.6 & 34.9 & 147.1 & 248.6 & 350.3 \\
& 50 & 16.2 & 198.2 & 367.5 & 527.4 & 49.1 & 229.2 & 387.1 & 542.4 \\
& 70 & 19.3 & 266.4 & 489.6 & 697.5 & 62.4 & 306.0 & 514.4 & 716.5 \\
\hline InSb & 1 & 3.8 & 10.7 & 18.5 & 25.9 & 6.2 & 13.0 & 20.0 & 27.0 \\
& 10 & 6.9 & 72.5 & 135.4 & 195.8 & 15.6 & 84.2 & 142.8 & 201.4 \\
& 30 & 12.3 & 192.1 & 349.7 & 494.4 & 42.2 & 218.7 & 365.6 & 506.1 \\
& 50 & 17.3 & 295.4 & 526.9 & 734.6 & 62.5 & 333.6 & 549.2 & 750.7 \\
& 70 & 22.0 & 387.1 & 680.7 & 940.3 & 81.2 & 435.2 & 708.4 & 960.2 \\
\hline GaAs & 10 & 32.0 & 46.1 & 66.5 & 83.6 & 42.1 & 56.1 & 72.4 & 88.1 \\
& 30 & 32.6 & 74.4 & 123.8 & 169.7 & 49.6 & 91.4 & 134.6 & 178.3 \\
& 50 & 34.3 & 103.7 & 181.0 & 254.5 & 57.3 & 126.6 & 195.9 & 266.5 \\
& 70 & 36.1 & 132.8 & 237.3 & 337.5 & 64.5 & 161.1 & 255.9 & 352.5
\end{tabular}

The present calculation has confirmed that the exponent of $r$ in exponential functions of all variational functions is a monotonically increasing function of the magnetic field from the value equal to 1 to a value less than 2 even for very high magnetic field. Energy differences between the $p m$ (Eq.(2)) and $d m$ (Eq.(3)) states for a given value $m>0$ listed in Table II are in very good agreement with experimental data for InAs [13, 14]. In the light of the present calculations all coulombic levels continuously rebuild their wave functions and continuously change their energies from the zero magnetic field to the high magnetic field values.

TABLE III

Band parameters used in the present calculations. $E_{0}$ denotes energy gap, $\Delta$ - spin-orbit splitting and $R y^{*}$ - effective rydberg.

\begin{tabular}{c|c|c|c}
\hline \hline Compound & $E_{0}[\mathrm{meV}]$ & $\Delta[\mathrm{meV}]$ & $R y^{*}[\mathrm{meV}]$ \\
\hline InAs & 418.0 & 380.0 & 1.4 \\
\hline InSb & 235.2 & 803.0 & 0.65 \\
\hline GaAs & 1519.2 & 341.0 & 5.76
\end{tabular}




\section{References}

[1] P. Löwdin, J. Chem. Phys. 19, 1376 (1951).

[2] C.R. Pidgeon, R.N. Brown, Phys. Rev. 146, 575 (1966).

[3] M.H. Weiler, R.L. Aggarwal, B. Lax, Phys. Rev. B 17, 3269 (1978).

[4] M. Brown, U. Rössler, J. Phys. C 18, 3365 (1985).

[5] J. Wlasak, J. Phys. C 19, 3459 (1986).

[6] R. Grisar, H. Wachernig, G. Bauer, J. Wlasak, J. Kowalski, W. Zawadzki, Phys. Rev. B 18, 4355 (1978).

[7] P. Pfeffer, W. Zawadzki, Phys. Rev. B, Condens. Maller 41, 1561 (1990).

[8] J. Wlasak, Acta Phys. Pol. A75, 61 (1989).

[9] D.M. Larsen, J. Phys. Chem. Solids 29, 271 (1968).

[10] W. Zawadzki, J. Wlasak, Theoretical Aspects and New Developments in Magneto-Optics, ed. I.T. Devrese, Plenum, New York 1980, p. 137.

[11] W. Trzeciakowski, M. Baj, S. Huant, L.C. Brunel, Phys. Rev. B, Condens. Matter 33, 6846 (1986).

[12] Y. Yafet, W. Keyes, E.N. Adams, J. Phys. Chem. Solids 1, 137 (1956).

[13] S.N. Holmes, R.A. Stradling, P.D. Wang, R. Droopad, S.D. Parker, R.L. Williams, Semicond. Sci. Technol. 4, 303, (1989).

[14] S.N. Holmes, R.A. Stradling, P.D. Wang, to be published in Semicond. Sci. Technol. 